\title{
Validation of Separation Accuracy for Small DNAs in a Capillary Gel Electrophoresis System with a Short-Length Fused Silica Capillary
}

\author{
Tomoka Nakazumi and Yusuke Hara \\ Research Institute for Sustainable Chemistry, ISC, National Institute of Advanced Industrial Science and Technology, \\ AIST, Central 5-2, 1-1-1 Higashi, Tsukuba 305-8565, Japan \\ Email: \{nakazumi.t, y-hara\}@aist.go.jp
}

\begin{abstract}
Despite the advantages of capillary gel electrophoresis (CGE) systems for point-of-care testing, they are limited by their large and complex equipment. Accordingly, we evaluated a scaled-down CGE system, we adopted a short-length capillary (total length of $15 \mathrm{~cm}$ and effective length of $7.5 \mathrm{~cm}$ ). The short-length capillary was coated with a nonionic-polymer chain to prevent electroosmotic flow with a direction of flow opposite to that of the DNA samples. We evaluated the electropherograms measured over 3 consecutive days by the CGE system with the short-length capillary under the same experimental conditions using a 100-bp DNA Ladder sample. Comparison of the electropherograms over time showed good stability and repeatability for migration time, mobility, and resolution length, demonstrating the suitability of this system for rapid and accurate point-of-care testing applications.
\end{abstract}

Index Terms - capillary gel electrophoresis, DNA, injection, gel, polymer solution

\section{INTRODUCTION}

The separation of small DNA fragments has attracted attention from the viewpoint of application to point-ofcare testing (POCT). Capillary gel electrophoresis (CGE) is considered a more suitable method for the separation of small DNAs compared to slab gel electrophoresis [1]-[5] owing to its speed, high resolution, and the small amount of DNA sample required. However, currently available CGE equipment is very large, expensive, and requires highly trained staff. These aspects have limited the application of CGE for analyzing pathogenic bacteria in a clinical setting, making conventional CGE unsuitable for POCT. To overcome this barrier, we took on the challenge of developing a compact CGE system for application to POCT in clinical settings. To scale down the CGE system, we adopted a short-length capillary to separate small DNA fragments. To use the fused silica capillary for the separation of small DNAs in the CGE process, the negatively charged capillary wall must first be treated because a bare capillary causes electroosmotic flow (EOF) running in the opposite direction of the DNA samples [6]-[9]. Indeed, DNA samples cannot be inserted

Manuscript received June 7, 2017; revised August 30, 2017 into the capillary when using a non-treated capillary due to EOF. Therefore, we selected an acrylamide polymer chain as the coating polymer on the capillary walls because it is a non-ionic polymer. In addition, to separate DNA samples using the capillary, the polymer solution should be inserted into the capillary as a molecular sieve [10], [11]. In this study, we selected a $0.5 \mathrm{w} / \mathrm{v} \%$ hydroxyethyl cellulose (HEC) solution with a molecular length of $1,300,000$.

We designed a CGE system consisting of a highvoltage power supply and a microscope with epiillumination. The evaluation of repeatability is an important step in the development of a self-built system with high accuracy. Therefore, in this study, we used our developed CGE system to obtain multiple measurements over 3 consecutive days by separating a 100-bp DNA Ladder sample. The electropherograms were evaluated according to migration time, mobility, and resolution length.

\section{EXPERIMENTAL SECTION}

\section{A. CGE Chemicals}

A 100-bp DNA Ladder (TAKARA BIO, Shiga, Japan) was utilized for this evaluation. This sample contained 11 double-stranded fragments with lengths of 100, 200, 300, 400, 500, 600, 700, 800, 900, 1000, and $1500 \mathrm{bp}$. The DNA ladder $(130 \mu \mathrm{g} / \mathrm{mL})$ was diluted 10 times using ultrapure water. HEC with a molecular size of $1,300,000$ was adopted as the sieving polymer solution. The running buffer was $0.5 \times$ Tris-borate-ethylenediaminetetraacetic acid buffer.

\section{B. Procedure for Coating the Fused Silica Capillary}

The fused silica capillary (Polymicro Technologies, Phoenix, AZ, USA) was washed using $1 \mathrm{~N} \mathrm{NaOH}$ (15 $\min )$, water (15 min), and methanol (15 min). In the next step, a solution containing $80 \mu \mathrm{L}$ of 3methacryloxypropyltrimethoxysilane (Shin-Etsu Chemical, Tokyo, Japan), $1 \mathrm{~mL}$ of methanol, and one drop of acetic acid was flowed into the washed capillary for $2 \mathrm{~h}$. In the final step, the monomer solution $(20 \mathrm{~mL})$ including the acrylamide monomer $(0.7 \mathrm{~g})$ and ammonium persulfate $(20 \mathrm{mg}), \quad N, N, N^{\prime}, N^{\prime}-$ 
tetramethylethylenediamine $(20 \mu \mathrm{L})$, which was bubbled with nitrogen gas for $30 \mathrm{~min}$, was flowed into the capillary for $2 \mathrm{~h}$.

\section{Self-Built CGE System}

The CGE system consisted of a high-voltage power supply (HJPQ-10P3; Matsusada, Osaka, Japan) and a microscope with epi-illumination (IX73; Olympus, Tokyo, Japan) (see Fig. 1). SYBR Green II was adopted to detect small DNA fragments based on fluorescence. The excitation light source was a mercury lamp that passed through an optical filter (U-FBWA; Olympus). A 60× objective lens (UPlanFLN; Olympus) and a photomultiplier tube (H8249-101; Hamamatsu Photonics, Hamamatsu, Japan) were used to detect SYBR Green II fluorescence from DNA samples. National Instrument NI USB-6341 was used to convert the fluorescence signal to digital data for quantification. The diameter of the fused silica capillary was $75 \mu \mathrm{m}$, and it was cut to a total length of $15 \mathrm{~cm}$ (effective length of $7.5 \mathrm{~cm}$ ). As the 100-bp DNA Ladder sample was injected into the capillary, 1.5 $\mathrm{kV}$ was applied to the sample solution for $1 \mathrm{~s}$. The DNA separation procedure was then performed by applying $100 \mathrm{~V} / \mathrm{cm}$.

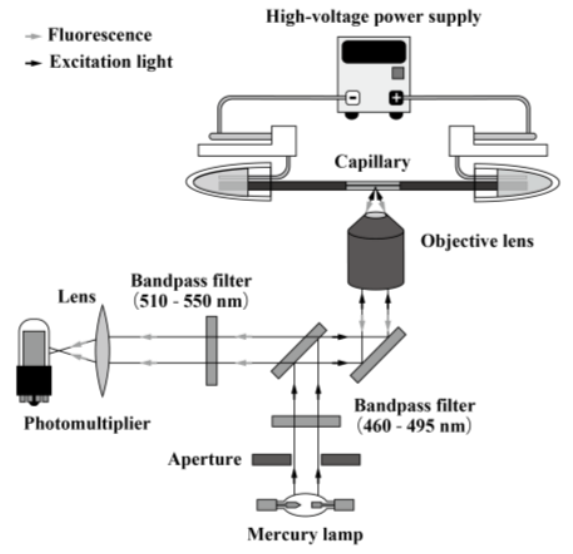

Figure 1. Schematic illustration of the self-built capillary gel electrophoresis system.

\section{RESULTS AND DISCUSSION}

Fig. 2 shows the electropherograms obtained using the self-built CGE system with HEC as the sieving polymer and a capillary coated with a non-ionic acrylamide polymer chain to prevent EOF. The self-built CGE system could clearly separate the 100-bp DNA Ladder sample using the short-length capillary. To determine the repeatability, we analyzed the relationship between the migration time or mobility and the double-stranded DNA size for electropherograms obtained over 3 consecutive days. As shown in Fig. 3, the migration time was consistent over time, with small error bars. Mobility refers to the transfer rate $(\mathrm{m} / \mathrm{s})$ of DNA divided by the applied voltage (V) per capillary length (m). The difference in the transfer rates of DNA fragments of different sizes are determined by the entanglement among the DNA samples and the sieving polymer solution. In general, when increasing the length of DNA, the entanglement strength increases, and it is this principle that allows for CGE to effectively separate DNA fragments of different lengths. As shown in Fig. 4, the error bars of the mobility did not vary over continuous measurements for 3 days, and the average values for each day were nearly identical (see Fig. 4(D)).

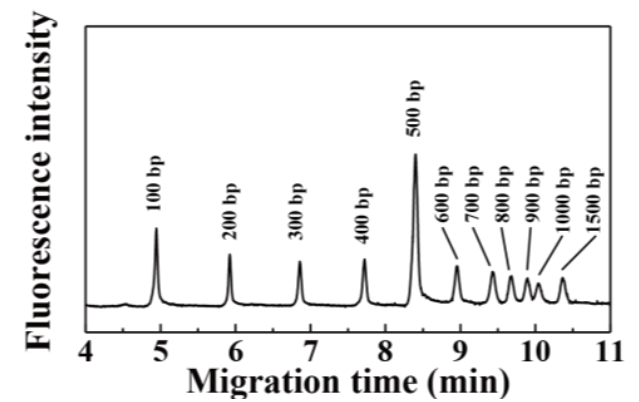

Figure 2. Separation of the 100-bp DNA Ladder into smaller DNA fragments using the self-built capillary gel electrophoresis system.

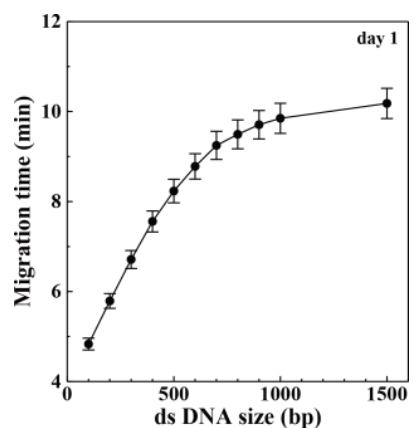

(A)

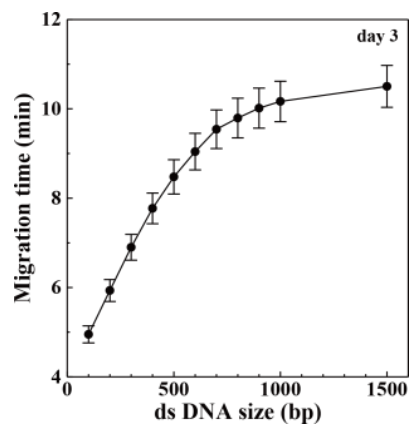

(B)

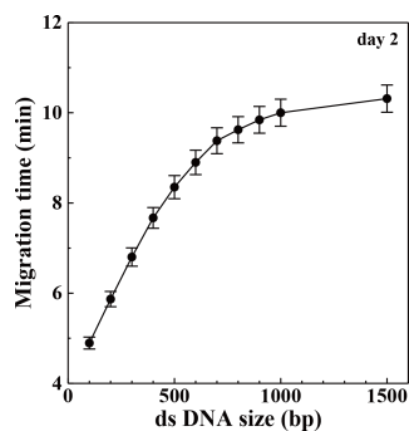

(B)

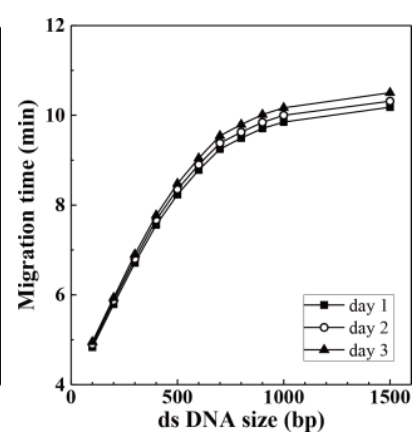

(D)
Figure 3. Relationship between migration time and dsDNA size (bp) measured over 3 consecutive days using the self-built CGE system. (A) day 1 , (B) day $2,(\mathrm{C})$ day 3 , and (D) average values for each day.

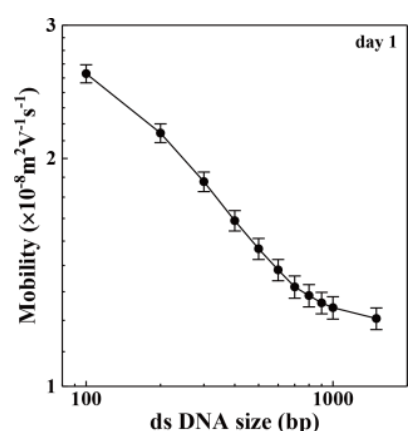

(A)

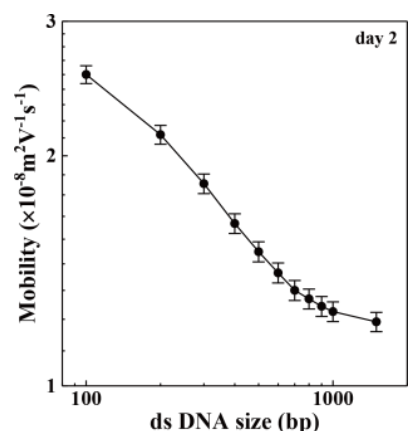

(B) 


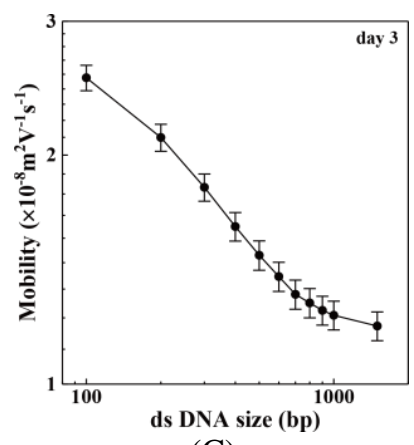

(C)

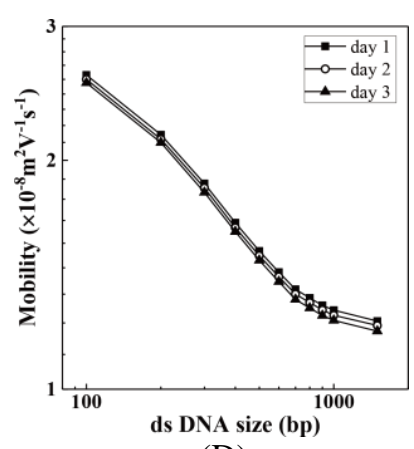

(D)
Figure 4. Relationship between mobility and dsDNA size (bp) measured over 3 consecutive days using the self-built CGE system. (A)

day $1,(\mathrm{~B})$ day $2,(\mathrm{C})$ day 3 , and (D) average values for each day.

Fig. 5 shows the relationship between the resolution length (RSL) and DNA size. To calculate the RSL, we calculated the resolution (Rs) of the electropherogram according to the following equation:

$$
\mathrm{Rs}=1.18 * \Delta t /\left(\mathrm{w}_{0.5}(1)+\mathrm{w}_{0.5}(2)\right)
$$

Where w0.5 is the full width at the half maximum of the peak, and $\Delta t$ is the difference in the migration time between two consecutive peaks in the electropherograms. The RSL can then be expressed by the following equation:

$$
\mathrm{RSL}=\Delta \mathrm{n} / \mathrm{Rs}
$$

Where Rs is the resolution value and $\Delta \mathrm{n}$ is the difference in the DNA length of adjacent peaks in the electropherogram. As shown in Fig. 5, the RSL did not change over the three measuring days. This trend indicated that our CGE system can accurately separate DNA using a short-length capillary coated with a nonionic polymer chain.

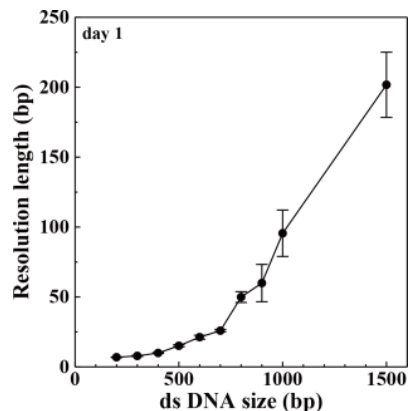

(A)

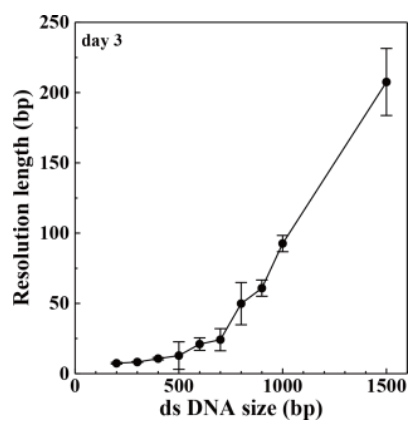

(C)

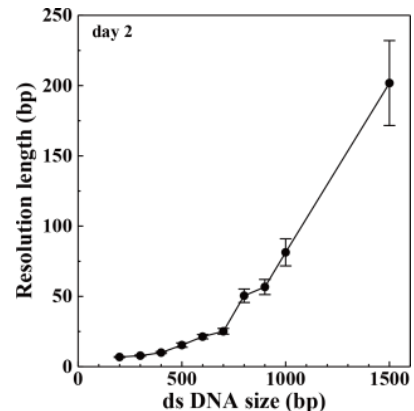

(B)

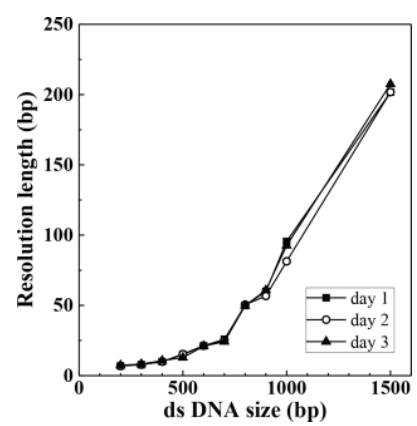

(D)
Figure 5. Relationship between resolution length and dsDNA size (bp) measured over 3 consecutive days using the self-built CGE system. (A) day $1,(\mathrm{~B})$ day $2,(\mathrm{C})$ day 3 , and (D) average values for each day.

\section{CONCLUSIONS}

In this study, we evaluated the ability of a self-built CGE system to separate a 100-bp DNA Ladder sample based on measurements obtained over 3 consecutive days for application to POCT. The electropherograms were analyzed according to migration time, mobility, and resolution length. The migration time and mobility are essentially determined by the entanglement strength between the DNA samples and the sieving polymer chain. Neither mobility nor migration time changed appreciably over 3 days. In addition, the RSL, calculated according to the resolution and the $\Delta \mathrm{n}$ to indicate the DNA lengthdifference of adjacent peaks, also did not change across measurement days. This trend indicates that the voltage application condition of our CGE system was significantly stable at each measurement point. In the next step of this research, we plan to scale down the CGE equipment further so that it does not require use of a microscope with epi-illumination and a mercury lamp. We aim to report this even more compact CGE equipment and demonstrate its application to POCT in the near future.

\section{ACKNOWLEDGMENT}

This work was supported by Grants-in-Aid (KAKENHI) for Young Scientists (B) (16K17493) and Scientific Research (B) (15H03827).

\section{REFERENCES}

[1] K. R. Mitchelson and J. Cheng, "Capillary Electrophoresis of Nucleic Acids Volume II: Practical Application of Capillary Electrophoresis," in Methods in Molecular Biology, vol. 163, New Jersey: Humana Press, 2001

[2] R. Higuchi, G. Dollinger, P. S. Walsh, and R. Griffith, "Simultaneous amplification and detection of specific DNA sequences," Biotechnology, vol. 10, p. 413, 1992.

[3] S. Furutani, N. Naruishi, M. Saito, E. Tamiya, Y. Fuchiwaki, and H. Nagai, "Rapid and highly sensitive detection by a real-time polymerase chain reaction using a chip coated with its reagents," Analytical Sciences, vol. 30, p. 569, 2014.

[4] P. G. Righetti, Capillary Electrophoresis in Analytical Biotechnology, Boca Raton, FL: CRC Press, 1996.

[5] A. S. Cohen, D. R. Najarian, A. Paulus, A. Guttman, J. A. Smith, and B. L. Karger, "Rapid separation and purification of oligonucleotides by high-performance capillary gel electrophoresis," Proceedings of the National Academy of Sciences of the United States of America, vol. 85, no. 2, p. 9660, 1988.

[6] J. Horvath and V. Dolník, "Polymer wall coatings for capillary electrophoresis," Electrophoresis, vol. 22, p. 644, 2001.

[7] D. Schmalzing, C. A. Piggee, F. Foret, E. Carrilho, and B. L. Karger, "Characterization and performance of a neutral hydrophilic coating for the capillary electrophoretic separation of biopolymers," Journal of Chromatography A, vol. 652, p. 149, 1993.

[8] C. Y. Liu, "Stationary phases for capillary electrophoresis and capillary electrochromatography," Electrophoresis, vol. 22, p. 612, 2001.

[9] C. S. Robb, "Applications of physically adsorbed polymer coatings in capillary electrophoresis," Journal of Liquid Chromatography and Related Technologies, vol. 30, p. 729, 2007.

[10] Y. Masubuchi, H. Oana, M. Matsumoto, M. Doi, and K. Yoshikawa, "Conformational dynamics of DNA during biased sinusoidal field gel electrophoresis," Electrophoresis, vol. 17, p. $1065,1996$. 
[11] H. Oana, Y. Masubuchi, M. Matsumoto, M. Doi, Y. Matsuzawa, and K. Yoshikawa, "Periodic motion of large DNA molecules during steady field gel electrophoresis," Macromolecules, vol. 27, p. 6061, 1994.

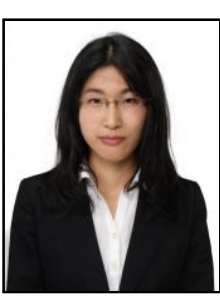

Tomoka Nakazumi received the B.S. degree from Hokkaido University, Hokkaido, Japan, in 2009, and the M.S. and Dr. Sci. degrees from the Tokyo Institute of Technology, Tokyo, Japan, in 2014. She is currently a Researcher with CleverMaterial Engineering Group, Research Institute for Sustainable Chemistry (ISC), National Institute of Advanced Industrial Science and Technology (AIST), Tsukuba, Japan. Her current research interests include photochemistry, polymer science, and soft actuators. Dr. Nakazumi is a member of the Chemical Society of Japan.

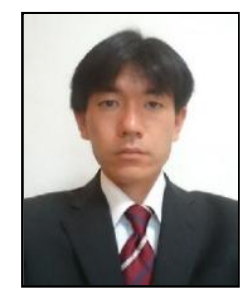

Yusuke Hara received the M.S. degree in the arts from Nagoya University, Nagoya, Japan, in 2001, and the Dr. Eng. degree in materials engineering from the Department of Materials Engineering, Graduate School of Engineering, University of Tokyo, Tokyo, Japan, in 2007. He is currently a Senior Researcher with CleverMaterial Engineering Group, Research Institute for Sustainable Chemistry (ISC), National Institute of Advanced Industrial Science and Technology (AIST), Tsukuba, Japan. From 2001 to 2007, he was researcher at the Research and Development Center, Lion Corporation, Tokyo. His current research interests include polymer science, soft actuator, and nonlinear chemistry. Dr. Hara is a member of the Chemical Society of Japan and the Society of Polymer Science, Japan. 